\title{
Multiple Extensive Complex Odontomas of the Jaws
}

\author{
MAHIMA V GULEDGUD ${ }^{1, *}$, SAIKRISHNA DEGALA ${ }^{2}$, KARTHIKEYA PATIL $^{1}$, DEEPIKA KESHARI ${ }^{3}$ \\ ${ }^{1}$ Professor, Department of Oral Medicine \& Radiology \\ ${ }^{2}$ Professor \& Head, Department of Oral \& Maxillofacial Surgery \\ ${ }^{3}$ Post Graduate Student, Department of Oral Medicine \& Radiology \\ *Corresponding author: dr.mahimavg@jssuni.edu.in
}

Received October 17, 2014; Revised October 20, 2014; Accepted October 23, 2014

\begin{abstract}
Odontomas are odontogenic tumors occurring in the oral cavity. They are composed of dental tissues namely enamel, dentin, cementum and pulp and are mostly discovered on routine radiographic examination owing to their benign and asymptomatic nature. Not many cases of odontomas erupting into the oral cavity are reported. An extensive survey of English literature revealed ten cases of erupted compound odontomas, and the present paper reports a case of a multiple symptomatic erupted complex odontomas of unusually large size involving both the jaws and the maxillary sinus in a 13-year-old male patient. Clinical and radiological examination using conventional and advanced diagnostic imaging with CT revealed an abnormally large radio-opaque mass, the density of which resembled enamel/bone occupying both the jaws and maxillary sinuses bilaterally. Diagnosis of odontoma was made which was confirmed histopathologically. Complete excision of the lesion with lining involving whole of maxillary sinus bilaterally and from the mandibular anterior region was done. Post operative period was uneventful with no evidence of recurrence.
\end{abstract}

Keywords: jaw, mandible, maxilla, maxillary sinus, multiple complex odontomas

Cite This Article: MAHIMA V GULEDGUD, SAIKRISHNA DEGALA, KARTHIKEYA PATIL, and DEEPIKA KESHARI, "Multiple Extensive Complex Odontomas of the Jaws." International Journal of Dental Sciences and Research, vol. 2, no. 6 (2014): 128-132. doi: 10.12691/ijdsr-2-6-2.

\section{Introduction}

The term odontoma was described by Paul Broca in 1867 to describe all odontogenic tumors and defined them as tumors formed by the overgrowth or transitory of complete dental tissue $[1,2,3,4]$. Odontomas are hamartomatous malformations rather than a true neoplasm consisting of ectodermal and mesodermal components. As they are composed of both cell types, they have been previously termed as "Composite Odontomas" [2].

Epidemiologically, odontomas account for $22 \%$ of all odontogenic tumors of the jaws [1,5]. The odontoma is a tumor of unknown etiology [6]. However, it has been attributed to different pathological conditions such as local trauma, inflammatory and/or infectious processes, mature ameloblasts, cell rests of Serres (dental lamina remnants), odontoblastic hyperactivity or due to hereditary anomalies like Gardener's syndrome and Hermann's syndrome, and alterations in genetic component responsible for controlling dental development $[7,8]$. They are formed due to budding of extra odontogenic epithelial cells originating from the dental lamina. They may exhibit expansile type of biologic behavior and may cause localized bone resorption, but does not invade adjacent tissue thus making their removal easy by curettage and conservative surgical management [6].

The second type of WHO Histologic typing of odontogenic tumors classifies odontomas under the broad category of tumors containing the odontogenic epithelium with odontogenic ectomesenchyme, with or without dental hard tissue formation. This classification has listed out three types of odontomas namely, odontoameloblastoma, complex and compound odontoma [3].

Based on location, odontomas have been classified as central or intraosseous, peripheral or extraosseous and erupted types [3,7]. The 2005 WHO classification of odontogenic tumors classifies odontomas as compound odontoma, complex and ameloblastic fibro-odontoma $[2,8]$. Compound odontomas consist of calcified tissues arranged in an organised manner bearing some resemblance to the normal teeth and occur most commonly in the anterior maxilla [67\%]. Complex odontomas consist of calcified dental tissues arranged as a disorganized, irregular mass bearing no morphologic similarities to rudimentary teeth and are found mostly in posterior mandible [1,2,3,8]. Compound odontomas are twice as common as complex variety [3]. Ameloblastic fibro-odontomas are composed of varying amounts of calcified tissue resembling an ameloblastic fibroma. They are considered as precursors of complex odontomas $[3,8]$.

Odontomas are developmental anomalies which are usually small in size ranging from few millimeters to 3-4 cms [9]. Very rarely they may exhibit an aggressive growth and become large enough to cause obstruction of the sinuses leading to morbidity [8].

Odontomas are mostly asymptomatic and discovered incidentally on radiographic examination. The clinical signs, indicative of odontomas include retained deciduous 
tooth, missing or impacted teeth [10]. However, occasionally they may become symptomatic causing swelling, pain or paraesthesia. Eruption of odontoma in the oral cavity is an extremely rare occurrence.

Although odontomas are common odontogenic tumors; multiple odontomas occur rarely in humans, particularly in association with some systemic syndromes such as familial adenomatosis polyposis [11].

\section{Case Report}

A 13 year old apparently healthy boy, reported to us with a chief complaint of an enlarging, painful swelling of upper jaw of one year duration along with nasal obstruction. His father gave history of trauma to the patient at the age of 5 years following which he lost his upper and lower anterior teeth. The swelling was insidious in onset, slowly growing and associated with moderate and intermittent pain. Past medical, dental and family histories were unremarkable. General physical examination revealed no systemic abnormalities.

Extraoral examination [Figure 1] showed a diffuse swelling over bilateral infraorbital region measuring approximately $2 \times 2 \mathrm{cms}$ extending from the ala of the nose to corner of the mouth, with normal appearing overlying skin, without any evidence of secondary changes. The swelling was non-tender, bony hard in consistency without any local rise in temperature. Intraoral examination revealed diffuse swelling in upper left palatal region extending from 65, anteriorly crossing the alveolar ridge to the depth of labial vestibule. In lower anterior region erupting hard tissue resembling a macrodont was noted associated with labial vestibular obliteration. Bilaterally upper anteriors and lower centrals and laterals were clinically missing [Figure 2]. Patient was subjected to routine radiological, hematological, biochemical and serological examination which showed elevated serum alkaline phosphatase (584.0 IU/L).

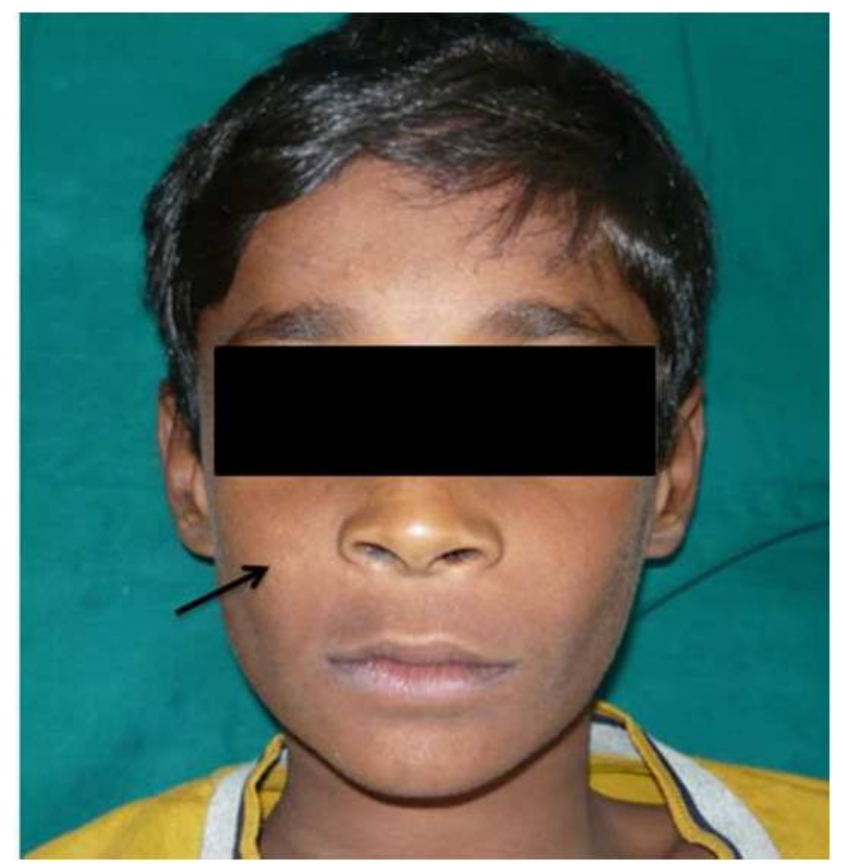

Figure 1. Pre-operative frontal profile showing diffuse swelling over bilateral anterior maxillary region

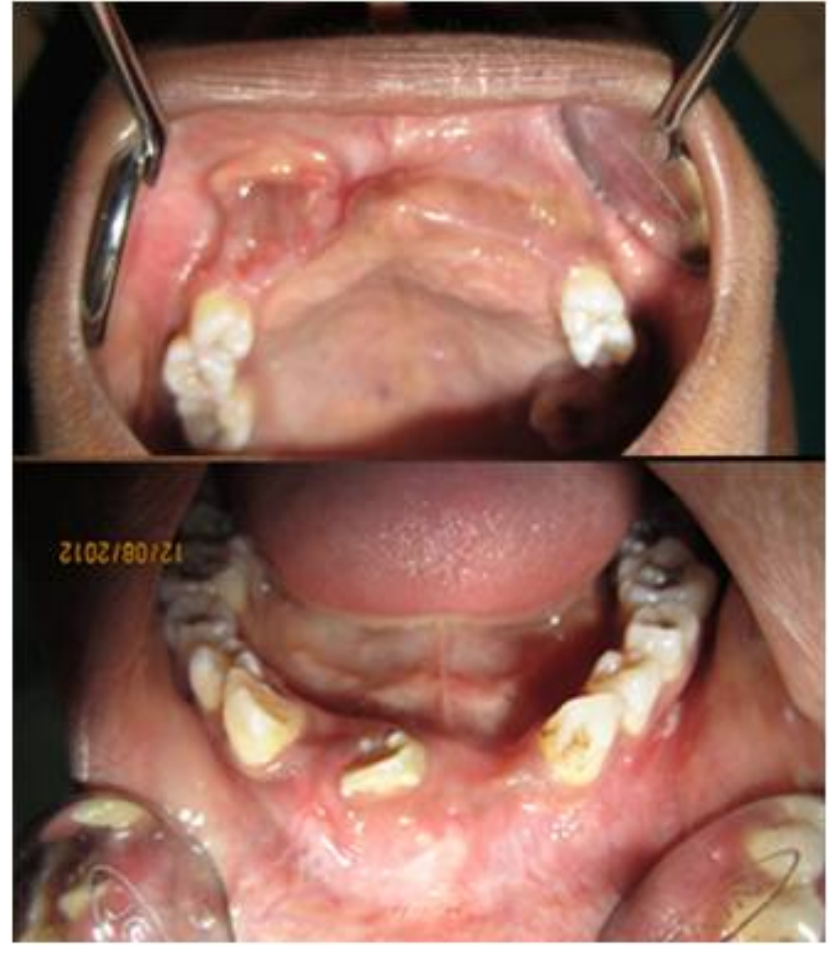

Figure 2. Intraoral photograph showing erupting mandibular odontoma and swelling in anterior maxillary alveolar ridge

Orthopantamograph [Figure 3] and maxillary and mandibular occlusal views showed irregular radio opaque structures in upper and lower anterior region, the density of which resembled bone/enamel. Intraoral periapical radiograph revealed diffuse radio opaque mass in the region of 12,11,21,22 and a well defined radio opaque mass lined by radiolucent rim in the region of 32,31,41,42. Computed tomography scan showed irregular hyperdense lesions (2000 HU) involving maxillary alveolar process on both sides extending to the floor of maxillary antrum measuring 3.8 X $2.6 \mathrm{~cm}$ on right side, 3.6 X $2.7 \mathrm{~cm}$ on left side. Adjacent permanent teeth were deformed. Well defined osteolytic lesion measuring 2.2 X $1.8 \mathrm{~cm}$ was noted involving mandible adjacent to symphysis menti [Figure 4]. Ossifying density (2000 HU) was noted within the osteolytic lesion. Adjacent incisor was deformed. The radiodense foci had attenuation values that were compatible with dentin (1000-1500 HU) and enamel ( $>1500$ HU) [12]. Considering the clinical and radiographic presentations, diagnosis of complex odontoma was made.

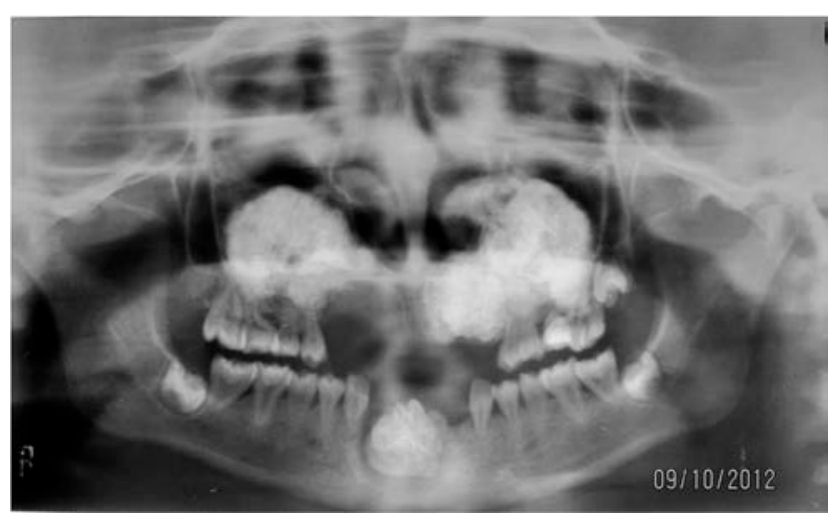

Figure 3. Pre operative Orthopantamograph 


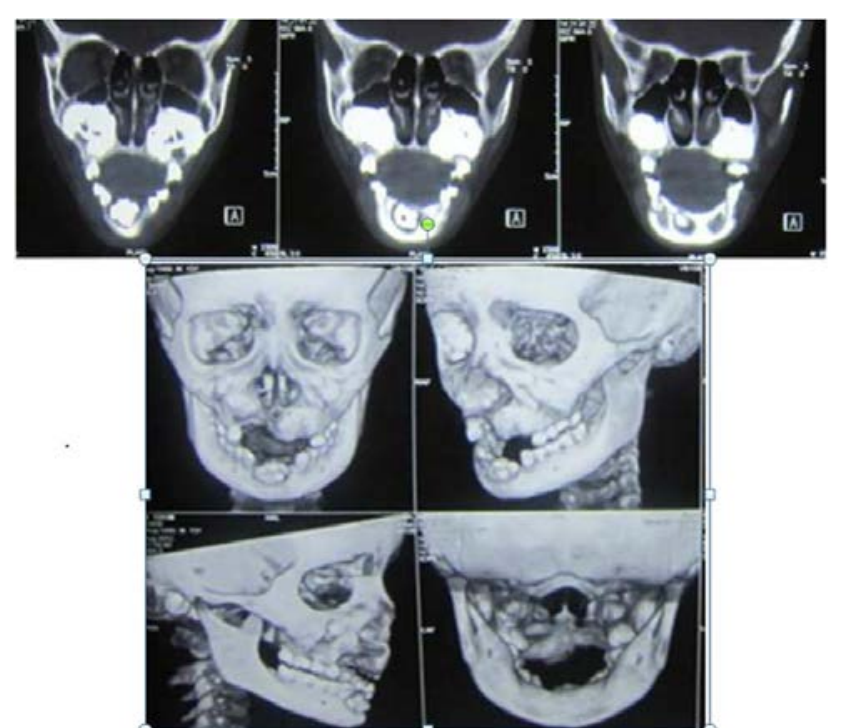

Figure 4. Pre operative CT scan showing hyperdense lesion involving maxillary sinus and in mandibular anterior region

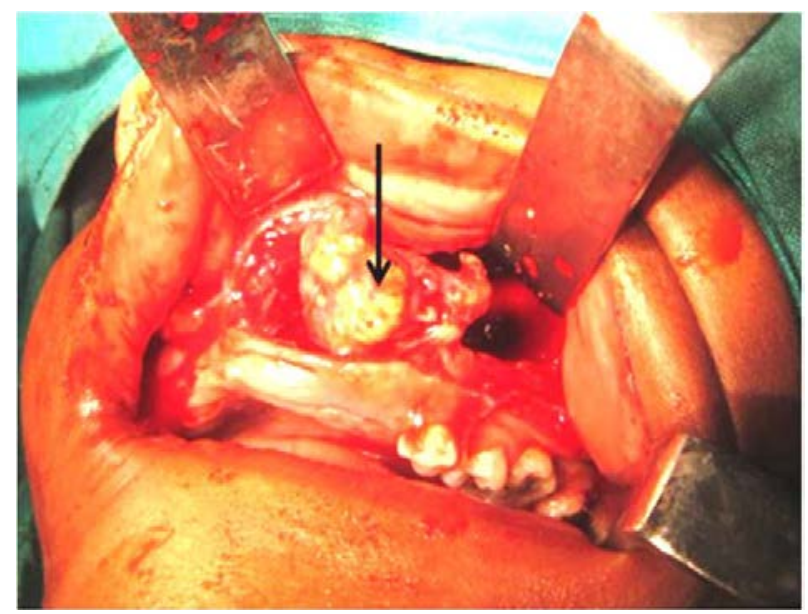

Figure 5. Intraoperative photograph showing macroscopic picture of odontoma being excised from left maxillary sinus

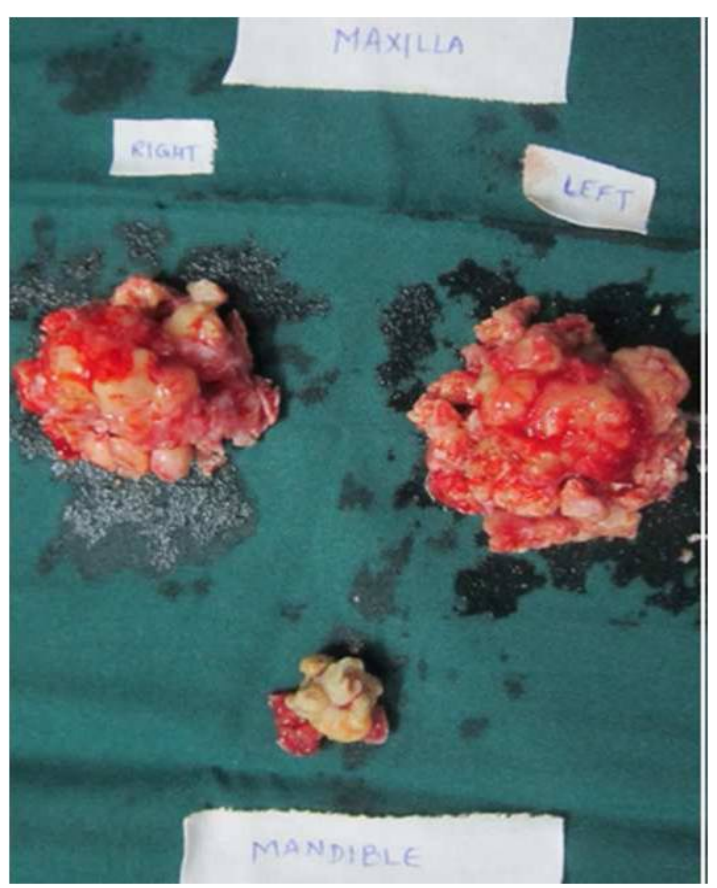

Figure 6. Photograph showing completely excised odontoma from both maxillary and mandibular region
Incisional biopsy was done under LA and the specimen sent for histopathological examination which showed masses of dysplastic dentin and fragments of enamel matrix. Clusters of eosinophilic ghost cells were seen suggestive of complex odontoma which confirmed the diagnosis. Surgery was performed under GA. Bilateral upper buccal vestibular and lower labial vestibular incision was placed, anterior maxilla exposed and odontoma was excised by breaking into multiple pieces using chisel and mallet owing to stony hard consistency of the lesion. Mandibular odontoma was enucleated in toto as the lesion was small and encapsulated. The maxillary lesion was found to involve entire maxillary sinus extending until floor of orbit [Figure 4, Figure 5, Figure 6]

After complete excision defect was curetted, irrigated, BIPPS pack was placed and primary suturing was done leaving behind a small window to remove the pack during post operative follow up. Specimen was sent for histopathological examination that revealed fragments of enamel matrix, masses of dentin and clusters of eosinophilic ghost cells [Figure 7].

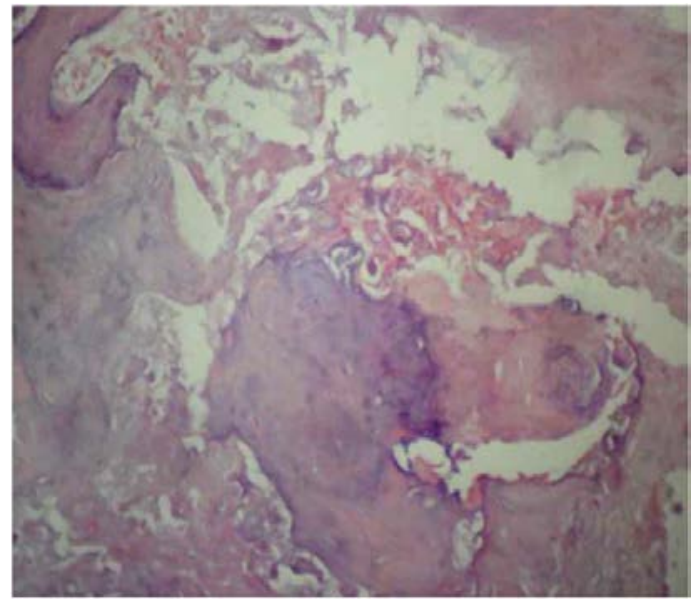

Figure 7. 20x photomicrograph showing ghost cells and dystrophic dentin

Post operative period [Figure 8, Figure 9, Figure 10, Figure 11] was uneventful; patient was under antibioitic coverage with nasal decongestants for a period of five days. One week later BIPPS pack was removed and sutured. Prosthetic rehabilitation of the patient was done. Patient is under follow up and no recurrences have been found.

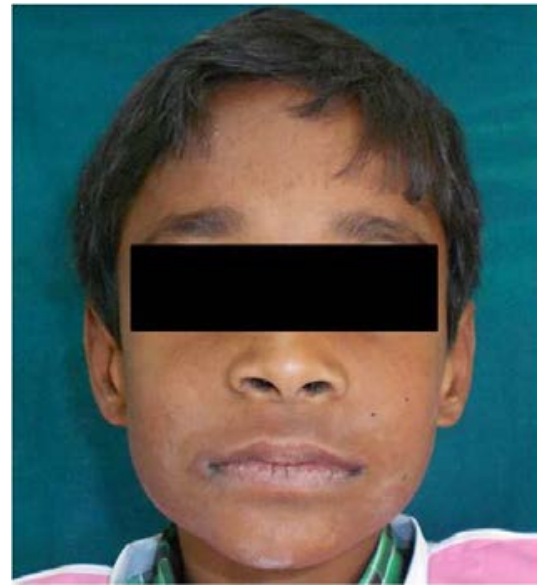

Figure 8. 2nd week post-operative frontal profile photograph 


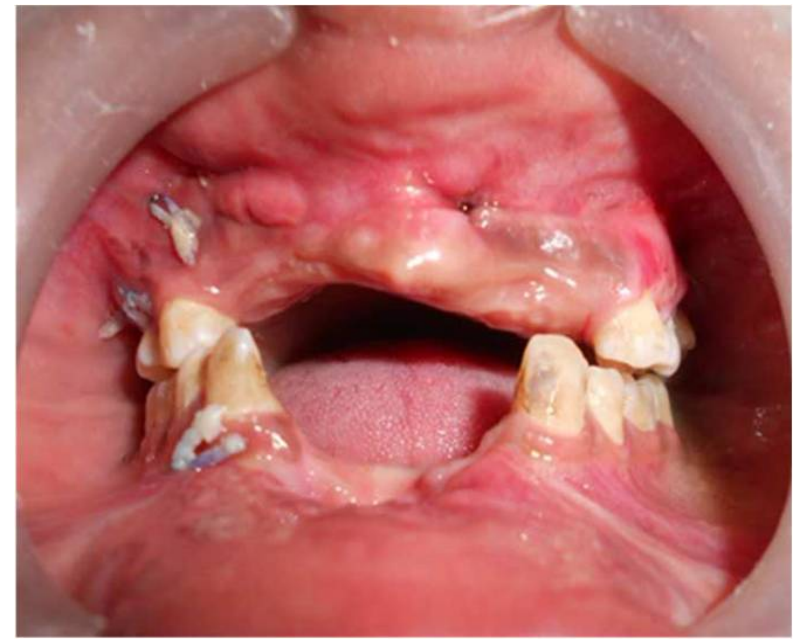

Figure 9. 2nd week Post-operative intra oral photograph

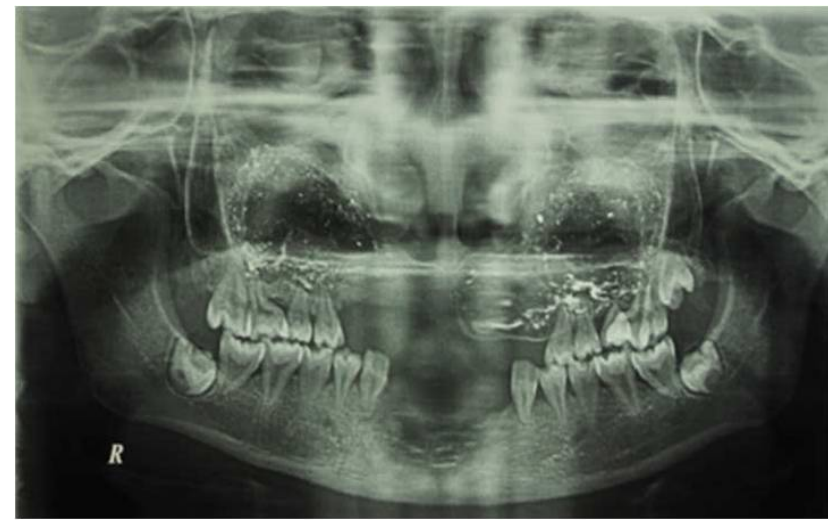

Figure 10. Post-operative orthopantamograph

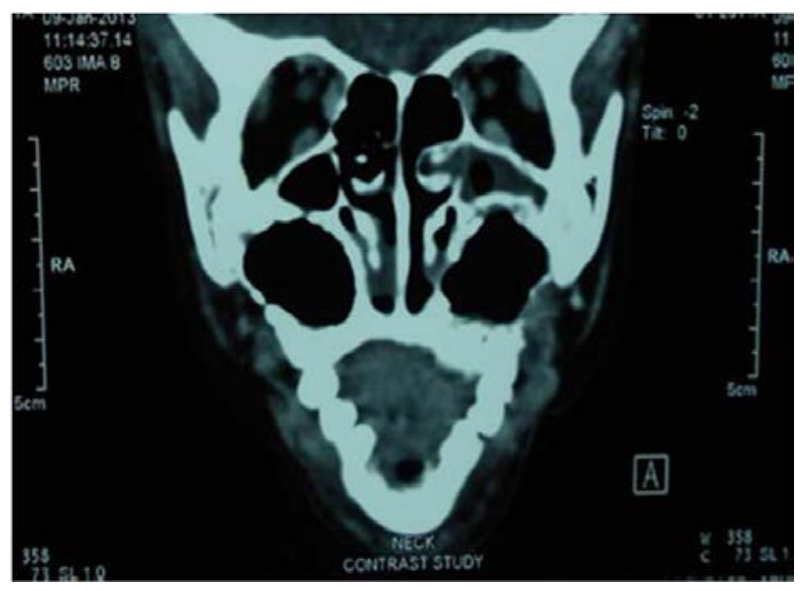

Figure 11. Post-operative CT coronal section

\section{Discussion}

Odontomas are relatively common odontogenic tumors, generally asymptomatic presenting with missing teeth leading to impaction or delayed eruption of permanent successor, displacement or ectopic eruption and malformation of adjacent teeth, anodontia and diastema. When symptomatic, odontomas present with pain, devitalization and resorption of tooth or bone due to the pressure exerted by the growing odontoma $[4,5,8]$. They may occur in any age; however, they are mostly diagnosed in the first two decades of life without any gender predilection $[4,5,13]$. Generally odontomas are small in size; however, occasionally they may grow large enough so as to cause bony expansion as seen in our case. They are thought to arise from budding of extra-odontogenic epithelial cells from the dental lamina. These clusters consisting of enamel, dentin, cementum and pulp may be deposited in an abnormal manner to form a large mass of tissues. Various factors such as prior trauma and infection appear to be involved, however, the exact etiology of odontoma formation is not known. Iturri et al reported a case of compound odontoma following trauma in the anterior face region in 10-year-old patient. Odontomas are more likely to occur if injury occurs early in childhood as concluded by a study conducted by Lopez Areal [4]. Odontoma like malformation affects permanent teeth following trauma to decidous dentition. It is described as a mass of calcified tissue showing slight structural resemblance to a normal tooth, having a relatively normal or rudimentary root. Our patient gave a history of trauma in his early childhood.

Gabell, James and Payne in 1914 classified odontoma arising from epithelial, composite (consisting of both epithelial and mesodermal components) and connective tissue origin according to development [1]. Thoma and Goldman in 1946 classified them as follows: Geminated composite odontomes, compound composite odontomes, complex composite odontomes, dilated and cystic odontomes [2,7]. In 1937, H.M. Worth grouped them as epithelial odontomes arising from dental epithelium (dentigerous cyst, dental cyst or multilocular cyst) and composite odontomes arising from the dental epithelium and dental mesoblestic tissues (complex, compound, geminated and dilated) [7]. Hybrid odontoma, a new variant has also been quoted in literature [2].

Eruption of the odontoma in the oral cavity is a rare occurrence with the first case being reported by Rumel et al in $1980[1,4,10]$. To the best of our knowledge, a total number of 21 cases of erupted odontomas have been reported in English literature, 10 of these being of compound variety and 11 of complex type. The reason for this type of behavior could possibly be due to the eruptive forces of the apparently impacted teeth and in cases of absence of teeth, may also be due to the resorption of alveolar ridge exposing the tumor, sequestration of overlying bone, remodelling of alveolar bone in case of young adults or reactive type of growth of the capsule around odontoma in elderly patients [7]. Erupted odontomas frequently result in pain and swelling followed by malocclusion with recurrent infections [1]. Compound odontomas are more frequent in the anterior region of the jaw whereas complex odontomas are more common in posterior jaw region. In the present case, odontoma was symptomatic, erupted, of complex type occupying anterior portion of both the jaws and whole of the maxillary sinus which is an extremely rare presentation. There is a possibility of obstruction of drainage of sinus when the tumor involves the sinus which may lead to complications such as orbital infections, epidural and subdural empyema, meningitis, cerebritis, cavernous sinus thrombosis, brain abscess and death [8]. However, our patient did not present with such symptoms.

The size of complex odontomas ranges from several millimeters to $3-4 \mathrm{~cm}$ [9]. Patil et al reported a case of large complex odontome involving the mandible and measuring about $5 \mathrm{~cm}$ in diameter in a $23 \mathrm{yr}$ old male 
patient [14]. Multiple odontomas with extensive jaw involvement is extremely rare in humans [15]. A literature review disclosed seven cases of multiple compound odontomas. Very few cases of multiple complex odontomas have been reported so far. Moreover, multiple complex odontomas with such extensive involvement of the jaw bones along with the maxillary sinuses bilaterally as seen in the present case is an extremely rare occurrence.

Radiographically, odontomas appear as well-defined radio-opaque mass, the density of which is greater than that of bone and greater than or equal to that of a tooth. A radiolucent halo, surrounded by a thin sclerotic line is noted, around the radiopacity. The radiolucent zone represents the connective tissue capsule of a normal tooth follicle and the corticated border of a normal tooth crypt is represented by the thin sclerotic line. The development of odontoma resembles the developmental stages of a normal tooth. In the initial stage, there is lack of calcification of the dental tissues making it to appear radiolucent. The intermediate stage is represented by partial calcification. The most radio-opaque stage is reached when the calcification of the dental tissues is complete $[3,8,16]$. In the present case additional radiographic evaluation with CT was required to determine the extent and features of the lesion as it allowed better depiction of the hyperdense mass approximating the floor of orbit.

The list of clinical differential diagnosis for complex odontoma includes adenomatoid odontogenic tumor, cementifying or ossifying fibroma, calcifying odontogenic cysts, calcifying epithelial odontogenic tumor and periapical cemental dysplasia [7]. Ameloblastic odontoma and ameloblastic fibroodontoma greatly resemble odontoma especially on radiograph [5].

Histologically, complex odontoma appears as an unorganized mass of hard odontogenic tissues while the compound odontoma resembles a normal tooth in its organisation. Odontogenic epithelial rests along with loose myxoid connective tissue may be found in close association with the lesion. Ghost cells, spherical dystrophic calcification, enamel concretions, and sheets of dysplastic dentin are also found [3]. Histological differential diagnosis includes ameloblastic fibroodontoma, odontoameloblastoma and calcifying odontogenic cyst.

Treatment consists of complete excision of the tumor along with associated soft tissues so as to prevent further complications like cyst formation, cystic degeneration or possible conversion to odonto-ameloblastoma [5]. In our patient there was no evidence of cyst formation. According to literature, once enucleated, odontoma usually does not recur; however, young children must be closely monitored for recurrences. In case of incomplete excision, the residual tumor does not show any changes [8].

\section{Conclusion}

Although benign and least known for aggressive behaviour, rarely odontomas may display unusual characteristics such as extensive size and multiple lesions. Pain, severe malocclusions, facial asymmetry and rarely sinusitis and brain abscesses due to involvement of the maxillary sinuses are a few complications encountered in such instances. Therefore, this paper has been written to throw light on the clinical and radiographic features of both the usual and unusual presentations, which would hasten the diagnosis and thereby aid in prompt treatment of the patient.

\section{Conflicts of Interest}

\section{None}

\section{References}

[1] Tejasvi A, Balaji M.L., Babu B. Erupted Compound Odontomas: A Case Report, Journal of Dental Research, Dental Clinics, Dental Prospects: 2011; 5, 33-36.

[2] Meetkamal and Kaur P. Odontoma Associated with NonSyndrome Multiple Supplemental Supernumerary Teeth, Journal of Clinical and Diagnostic Research, 2011: 5 (1): 142-145.

[3] Patil S, Rahman F, Tipu S.R., Kaswan S. Odontomas: Review of literature and report of case. Oral and Maxillofacial Pathology Journal, 2012: 3 (1); 224-227.

[4] Dua N, Kapila R, Trivedi A, Mahajan S, Gupta SD. An unusual case of erupted composite complex odontoma. Journal of Dental Sciences and Research 2011: 2 (2): 1-5.

[5] Das UM, Vishwanath D, Azhar U. A Compound Composite Odontoma Associated with Unerupted Permanent Incisor: A case report. International journal of clinical pediatric dentistry. 2009: 2 (2); 50-55.

[6] John JB, John RR, Punithavathy, Elango I. Compound Odontoma Associated with Maxillary Primary Tooth- A Case Report. JIADS 2010; 1 (1): 49-51.

[7] Arunkumar KV, V, Garg N. Surgical management of an erupted complex odontoma occupying maxillary sinus- case report. Ann Maxillofac Surg 2012; 2: 86-9.

[8] Srivastava A, Annaji AG, Nyamati SB, Singh G, Shivakumar GC, Sahana S. Complex odontoma in both the jaws: A Rare Case Report. J Orofac Res 2012; 2 (1):56-60.

[9] Carvalho CH, Costa DA, Queiroz L.M., Amaral J.I., Germano A.R.Extensive complex odontoma in the maxillary sinus: An uncommon presentation as a cause of chronic sinusitis. Rev Odonto Cienc 2011; 26 (1): 92-95.

[10] Isler SC, Demircan S, Soluk M, Cebi Z. Radiologic evaluation of an unusually sized complex odontoma involving the maxillary sinus by cone beam computed tomography. Quintessence international, 2009: 40 (7): 533-535.

[11] Matsuo K, Yamamoto K, Morimoto Y , Yamashita Y, Zhang M, Ishikawa A et al, Multiple complex odontomas and subsequent occurrence of an ossifying fibroma at the same site as the removed odontoma. Journal of Dental Sciences (2013) 8, 189-195.

[12] Shetty D C, Urs A B. Ameloblastic fibro-odontoma case report: diagnostic valuable aid of CT scan in identification of mineralized component. Journal of Indian Academy of Oral Medicine, 2010; 22 (2): 102-104.

[13] Serra-Serra G, Berini-Aytes L, Gay-Escoda C. Erupted odontomas: A report of three cases and review of literature. Med Oral Patol Oral Cir Bucal. 2009 Jun 1: 14 (6): E299-303.

[14] Patil K, Guledgud MV, Gupta B. Complex Odontome Causing Facial Asymmetry. Journal of Indian Academy of Oral Medicine and Radiology 2006, 18: 02, 134-137.

[15] Bordini J, Contar CM, Sarot JR, Fernandes A, Machado MA. Multiple Compound Odontomas in the Jaw: Case Report and Analysis of the Literature. J Oral Maxillofac Surg, 2008, 66: 2617-2620.

[16] Kavitha N, Venakateswarlu M and Geetha P. Radiological Evaluation of a Large Complex Odontoma by Computed Tomography. Journal of Clinical and Diagnostic Research, 2011; 5 (6): 1307-1309. 